\title{
Oligolectisme et décalage phénologique entre plante hôte et pollinisateur : étude de deux espèces printanières psammophiles, Colletes cunicularius (L.) (Hymenoptera, Colletidae) et Andrena vaga (PANZER) (Hymenoptera, Andrenidae)
}

\author{
Par Maryse Vanderplanck *, Etienne Bruneau ** et Denis Michez *
}

\begin{abstract}
Colletes cunicularius (L.) and Andrena vaga (Panzer), both vernal solitary ground nesting bees, have been reported to feed strictly on Salix (Salicaceae) (oligolectic behavior). However a recent study has shown that Colletes cunicularius is able to shift on different plants, at least in the studied sites in Germany. In this study we investigated the extent to which this behavior applies to other regions within the distribution range of these bee species. We analysed the host-plant association of two populations in Belgium. Pollen loads and brood cells of both species have been investigated by optical microscopic analysis to estimate the proportion of willow pollen. Andrena vaga females collect pollen only on Salix but pollen loads of Colletes cunicularius sometimes contain other pollen types in agreement with a previously study. It is observed that the first nest cells made by C. cunicularius in early spring contain exclusively willow pollen. Towards the end of willow blooming, Colletes cunicularius females change their floral choice by foraging on other pollen types.
\end{abstract}

Résumé. Colletes cunicularius (L.) et Andrena vaga (PANZER), deux espèces solitaires printanières psammophiles, ont été qualifiées d'oligolectiques strictes sur Salix (Salicaceae). Cependant des observations relativement récentes ont démontré le caractère polylectique de Colletes cunicularius, au moins pour la station étudiée en Allemagne. Il reste maintenant à déterminer quelle est la variation géographique de ce caractère. Pour ce faire, nous avons étudié deux populations de cette espèce en Belgique. Des charges de pollen de femelles et des cellules larvaires de ces deux espèces ont été analysées au microscope optique afin d'estimer les proportions de pollen de saule. Nos résultats montrent que les femelles d'Andrena vaga collectent du pollen uniquement sur Salix tandis que les charges de pollen de Colletes cunicularius contiennent parfois d'autres types de pollen. On observe que les premiers pains de pollen réalisés par les femelles de $C$. cunicularius au début du printemps contiennent exclusivement du pollen de saule. Lorsque les fleurs de saule se font plus rares, les femelles de Colletes cunicularius modifient leurs choix floraux en récoltant d'autres types de pollen.

Mots-clés. Colletes cunicularius, Andrena vaga, Salix, oligolectisme, changement d'hôte.

\section{Introduction}

Parmi les 16.000 espèces d'abeilles décrites (Michener 2007), certaines arborent une spécialisation dans leur choix de pollen, visitant un nombre restreint de plantes disponibles dans leur habitat (monolectisme, oligolectisme) tandis que d'autres butinent un plus large éventail de plantes hôtes (mésolectisme, polylectisme) (Robertson 1925; Cane \& Sipes 2006). Ces interactions plante-abeille ont déjà fait couler beaucoup d'encre et ont été le centre de nombreuses discussions. Divers auteurs ont ainsi tenté de classifier les différents types d'exploitation des ressources alimentaires observés au

* Maryse Vanderplanck \& Denis Michez, Laboratoire de Zoologie, Université de Mons, Place du Parc 20, B-7000 Mons, Belgique.

Email:marysevdp@hotmail.com,denis.michez@umh.ac.be

** Etienne Bruneau, Centre Apicole de Recherche et d'Information (CARI), 4 Place Croix du Sud, B-1348 Louvain-la-Neuve, Belgique. Email: bruneau@cari.be sein des Apoidea Apiformes (Robertson 1925; Rasmont 1988; Cane \& Sipes 2006; Müller \& Kuhlmann 2008).

Pour les abeilles, l'alimentation des larves et des imagos est entièrement basée sur la récolte de pollen et de nectar (Michener 2007). Contrairement aux espèces eusociales (à quelques exceptions près comme Bombus consobrinus et Bombus gerstaeckeri; Ponchau et al. 2006), les abeilles solitaires, au cycle de vie plus éphémère, sont le plus souvent oligolectiques. Pour ces espèces oligolectiques, il est primordial que l'émergence des imagos et la période de floraison de leur plante hôte soient synchronisées (Michez et al. 2008). Tout changement phénologique doit être corrélé avec un changement d'hôte(s) (Larkin et al. 2008).

$\mathrm{Au}$ cours de cette étude, nous nous sommes intéressés au degré de spécialisation de deux espèces printanières 

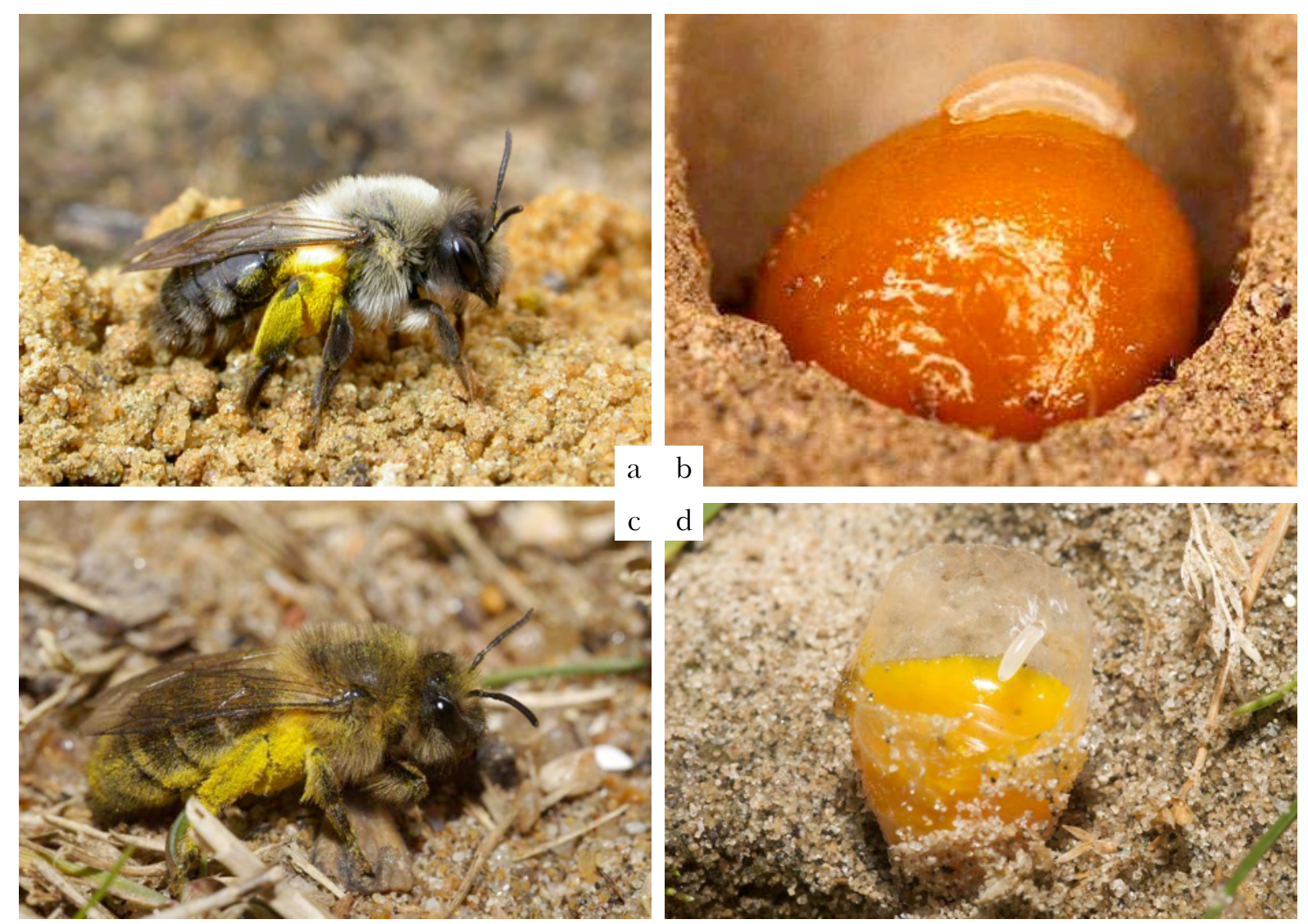

Figure 1. Espèces examinées dans le cadre de cette étude. a. Femelle d'Andrena vaga les pattes chargées de pollen; b. Détail d'une cellule larvaire d'Andrena vaga; c. Femelle de Colletes cunicularius les pattes chargées de pollen; d. Détail d'une cellule larvaire de Colletes. (Photos NJ Vereecken).

psammophiles : Andrena vaga (PANZER) (Hymenoptera, Andrenidae) (Figure la) et Colletes cunicularius (L.) (Hymenoptera, Colletidae) (Figure 1c). Ces abeilles solitaires nidifient généralement en grand nombre sur des espaces restreints. Ces agrégations peuvent comporter plusieurs centaines de nids en activité (Westrich 1989; Bischoff 2003; Vereecken et al. 2006) ce qui facilite la collecte de plusieurs cellules larvaires en peu de temps.

Chez Colletes cunicularius, les entrées des nids sont facilement reconnaissables grâce à l'accumulation de sable issu du creusement de la galerie par la femelle (tumulus) (Figure 2a). Une galerie principale débouche sur des loges larvaires ovales oblongues, indépendantes, tapissées d'une enveloppe (cellophane) protectrice et imperméable (Vereecken et al. 2006) (Figure 1d). Ces cellules sont approvisionnées en pollen et en nectar par les femelles.

Bien que l'architecture du nid d'Andrena vaga soit proche de celle observée chez Colletes cunicularius (Malyshev 1936), l'entrée des nids est obturée entre deux approvisionnements dans le cas des andrènes, ce qui rend leur repérage plus difficile (Figure 2 b). Les loges larvaires sphériques, exemptes de cellophane, abritent une pâte nourricière composée d'un mélange de nectar et de pollen (Figure 1b).

En Belgique, ces deux espèces univoltines comptent parmi les premières abeilles sauvages à émerger au cours de l'année (Vereecken et al. 2006). Les femelles des deux espèces présentent des différences au niveau de leur rythme d'activité. Grâce à sa taille plus grande et sa pilosité thoracique plus importante, Colletes cunicularius bénéficie d'une meilleure thermorégulation et commence à collecter plus tôt au matin (Bischoff et al. 2003). Généralement, elles partagent les mêmes exigences écologiques et sont couramment observées en syntopie (Vereecken et al. 2006). De ce fait, elles ont à disposition les mêmes ressources florales. Bien que les deux espèces aient été considérées comme strictement oligolectiques sur Salix, des observations suggèrent fortement que Colletes cunicularius visite des fleurs autres que celles du saule (Bischoff et al. 2003; Müller \& Kuhlmann 2008). Cependant, les analyses fines des choix floraux sont limitées géographiquement. L'évaluation claire de leur spécialisation a été menée ici en Belgique pour la première fois par le biais d'une analyse quantitative des charges de pollen et des ressources alimentaires accumulées dans les cellules larvaires.

\section{Matériel et Méthode}

Les observations ont été réalisées en avril 2008 sur le site de la Grande-Bruyère de Blaton (Belgique, Hainaut, WGS84 50²9'30.2'” 0340'25.5'E ca 56.4m) (Figure 3). Ce vaste site, altéré par le déversement de déchets et la fréquentation par les engins tout-terrains, bénéficie depuis peu du statut de réserve naturelle domaniale. Il est exceptionnel par la présence de nombreuses espèces végétales et animales protégées (Barone 1999). 

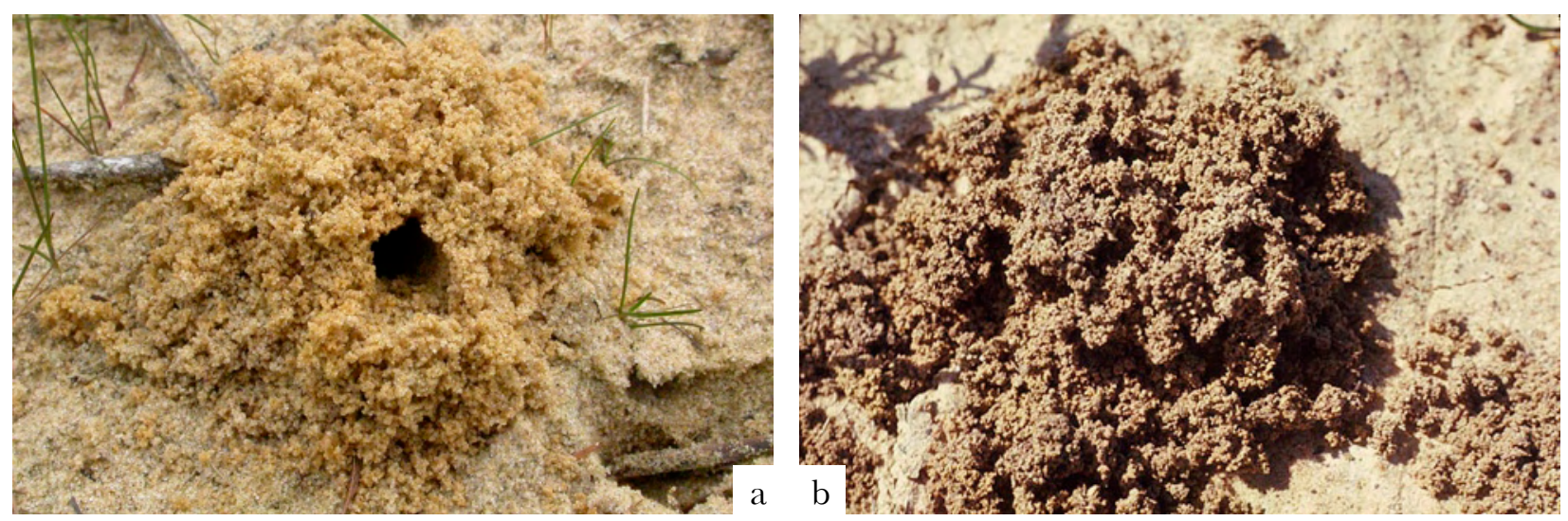

Figure 2. Détail de l'entrée du nid. a. Colletes cunicularius; b. Andrena vaga (Photos NJ Vereecken).

Il comprend notamment des étendues de sables non ou peu fixés, colonisées par une végétation caractéristique, des lambeaux de lande à callune colonisés par les genêts à balais et les bouleaux, des zones densément envahies de saules, quelques petits massifs boisés ainsi qu'une zone humide. En outre, on y retrouve en moindre proportion cerisier, tussilage, luzule, cardamine et pissenlit. L'intérêt entomologique de ce site est exceptionnel, notamment par la présence d'une très grande diversité hyménoptérologique dont certaines espèces protégées. Deux stations ont été établies, la première au sein même du site de la Grande Bruyère de Blaton où le saule était en fin de floraison et la seconde plus aux abords avec un effectif de saule en fleurs plus important.

Pour les analyses de pollen, dix femelles de chaque espèce avec charges de pollen ont été capturées au filet. Cet échantillonnage a été complété par dix cellules larvaires excavées de leurs nids respectifs à l'aide d'une bêche. Les différents échantillons ont été isolés dans des tubes eppendorfs et placés au congélateur à $-40^{\circ} \mathrm{C}$. Une fraction aléatoire de chaque échantillon $(n=40)$ a été étudiée par analyse au microscope optique à un grossissement de 256 fois (REICHERT) afin de déterminer les ressources de pollen des différentes espèces. Les pattes postérieures de chaque abeille ainsi que les réserves polliniques de chacune des cellules larvaires ont été tamponnées à l'aide d'un petit cube de gélatine disposé entre lame et lamelle.

Les échantillons ont été analysés par le laboratoire du centre apicole de recherche et d'information (CARI asbl) situé à Louvain-La-Neuve en Belgique (E. B.). Un balayage systématique des lames a été réalisé afin de dénombrer et d'identifier les différents pollens présents, cela afin d'établir le pourcentage de pollen des différentes espèces présentes. Les pourcentages sont moyennés pour tous les échantillons de chacune des espèces.

\section{Résultats}

\section{Observations comportementales}

Alors que la collecte s'est effectuée fin de matinée et début d'après-midi les 17 et 18 avril 2008, période de la journée où les deux espèces devraient être en pleine phase de récolte (Bischoff et al. 2003), une différence d'activité a pu être observée entre les femelles des deux espèces. Pendant que Colletes cunicularius volait en tout sens avec les scopae remplies de pollen, les andrènes restaient inertes au sol, exemptes de toute charge de pollen. Cette absence d'activité n'a été observée qu'au sein de la première station et semble être corrélée avec la fin de floraison du saule. En effet, là où la plante hôte était en pleine floraison, les femelles d'Andrena vaga effectuaient de nombreux voyages, collectant le pollen en grande quantité.

\section{Analyses palynologiques}

Toutes les cellules de Colletes cunicularius analysées $(\mathrm{n}=10)$ sont exclusivement composées de Salix sp. pur $(100 \%)$. Par contre, des types de pollen autres que le saule ont été trouvés dans les charges transportées par les femelles à un moment plus avancé de leur phénologie (Figure 4). La Figure 5 illustre le détail de l'analyse du pollen transporté par les différentes femelles collectées : les charges de deux femelles contiennent une majorité de pollens d'arbres fruitiers cf. Prunus sp. $(>99 \%)$ et une charge contient à la fois des pollens de Salix sp. (Figure 3a) et de cf. Prunus sp. (Figure 4 c) dans une proportion 80:20. Le pourcentage moyen de ce pollen fruitier étant supérieur à $4 \%$ (Figure 4 indiqué par $\left(^{*}\right)$ ), il ne s'agit pas d'un dépôt accidentel de ce pollen entomophile sur les fleurs de saule par le vent ou d'autres insectes, ce pollen a donc été collecté de manière active par l'abeille (Müller 1996). Les sept charges investiguées restantes contiennent majoritairement du Salix sp. et d'autres pollens en trace tels que le bouleau (Betula pendula), le tussilage (Tussilago farfara) et le pissenlit (Taraxacum sp.) considérés comme contaminants $(<1 \%)$ (Figure 5).

Les charges de pollen transportées par les femelles d'Andrena vaga contiennent une nettement moindre proportion de pollens étrangers par rapport à Colletes cunicularius. Dans six des dix charges analysées, nous avons trouvé des traces d'autres types de pollen à l'état isolé : Prunus sp., Betula pendula (Figure 3 b) et du Tussilago farfara. Vu leur très faible densité (1 à 2 sur 10.000), on peut considérer cela comme une contamination. Les quatre autres charges sont 


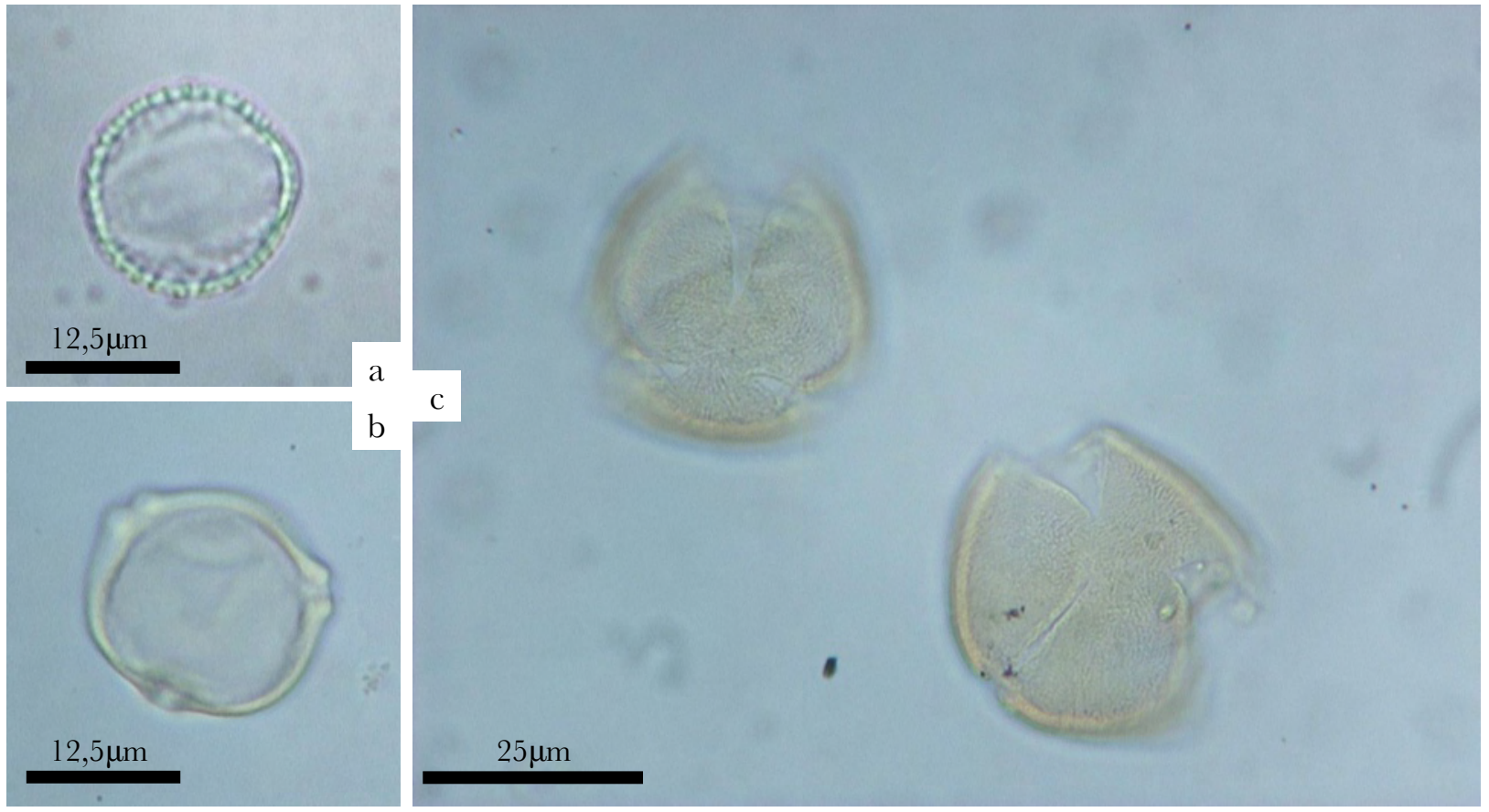

Figure 3. Exemples de pollens de référence sur lames. a. Pollen de saule (Salix sp.); b. Pollen de bouleau (Betula pendula) et c. pollen de cerisier (Prunus avium). (Photos CARI).

composées de Salix sp. pur. En ce qui concerne l'analyse des pains de pollen, outre les contaminants (bouleau, tussilage, pissenlit, cardamine (Cardamine pratensis) et Fabaceae), ils se composent exclusivement de pollen de saule. Les femelles d'Andrena vaga récoltent donc exclusivement du Salix sp. quelque soit la période de leur phénologie considérée.

\section{Discussion}

L'analyse microscopique et la comparaison qualitative des divers types de pollen collectés par les deux espèces confirment une différence significative entre leurs ressources alimentaires d'Andrena vaga et
Colletes cunicularius déjà observée par Bischoff (2003). Alors qu'Andrena vaga collectait quasiment du saule pur, d'autres types de pollen ont été retrouvés dans les charges de Colletes cunicularius. Leurs proportions suggèrent que les femelles de Colletes cunicularius collectent régulièrement du pollen sur diverses plantes hôtes autres que le saule (voir aussi l'article de Mahé (2009) dans ce numéro). De cette étude, nous confirmons aussi que le pourcentage de pollens autres que le saule collectés par Colletes cunicularius augmente au cours de la phénologie de l'insecte. En effet, lors de la construction du nid, les femelles collectaient uniquement sur le saule en pleine floraison comme en témoigne l'analyse des cellules larvaires. Par contre, vers

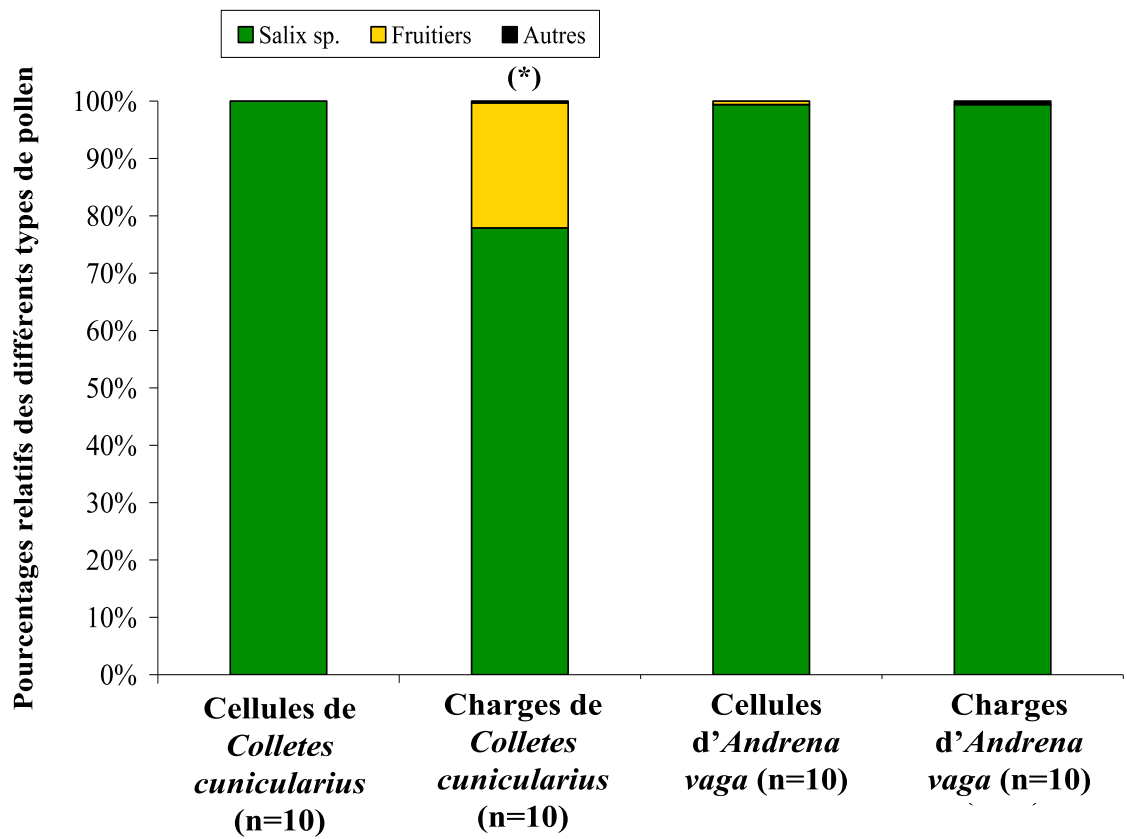

Figure 4. Pourcentage numérique relatif moyen du pollen de Salix sp. et des autres types de pollen dans les cellules larvaires excavées ( $\mathrm{n}=10$ ) et les charges des femelles $(n=10)$ pour chacune des deux espèces collectées en 2008. La catégorie autres reprend les types de pollen présents en trace et en proportions variables: Betula sp., Tussilago sp., Taraxacum sp., Cardamine et Fabaceae. L'astérisque $\left(^{*}\right)$ indique la série d'échantillons pour laquelle la proportion de pollen autre que le saule est significative (> $4 \%)$ 


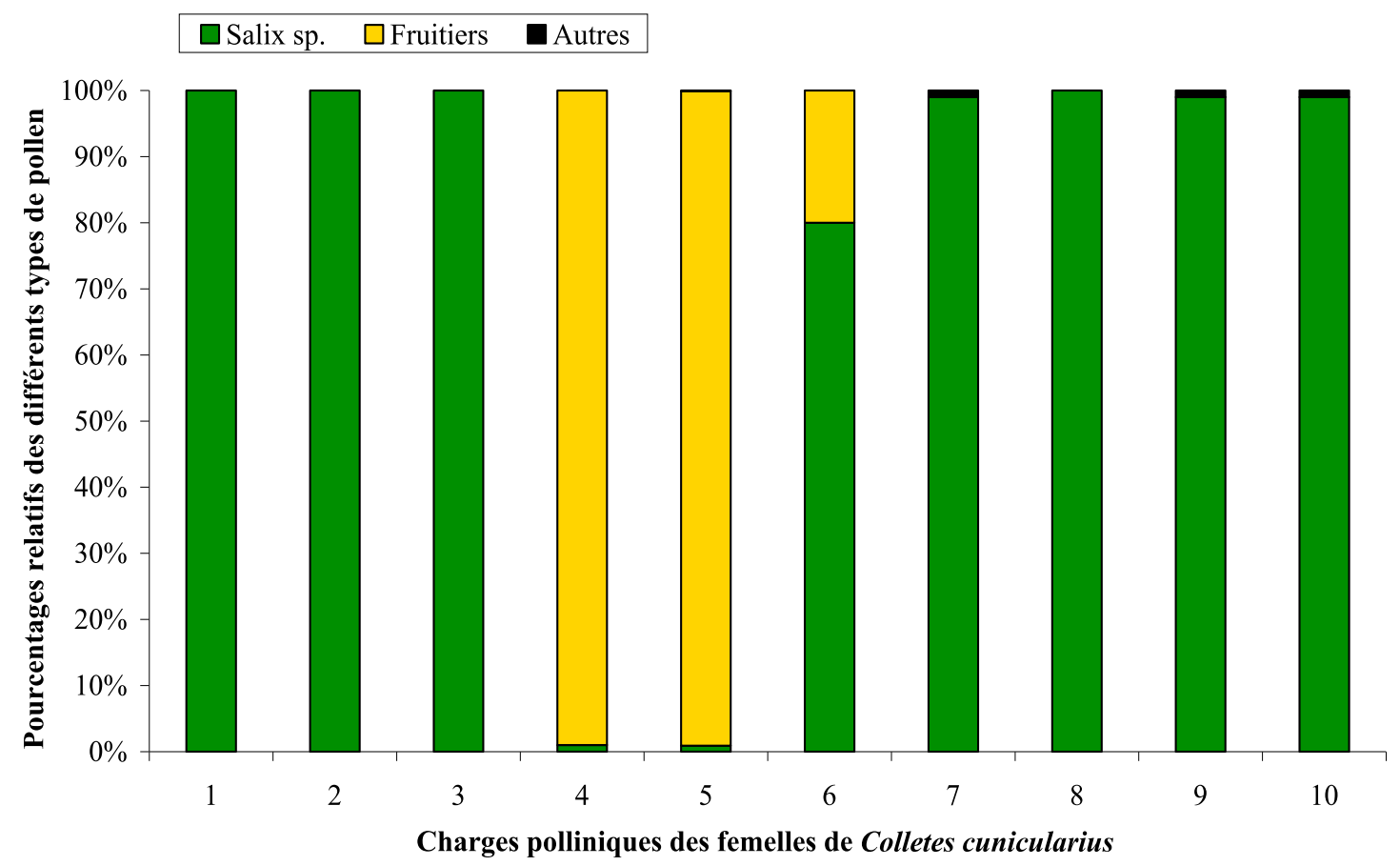

Figure 5. Pourcentage numérique relatif moyen du pollen de Salix sp. et des autres types de pollen dans les cellules larvaires excavées ( $\mathrm{n}=10$ ) et les charges des femelles $(n=10)$ pour chacune des deux espèces collectées en 2008. La catégorie autres reprend les types de pollen présents en trace et en proportions variables: Betula sp., Tussilago sp., Taraxacum sp., Cardamine et Fabaceae. L'astérisque (*) indique la série d'échantillons pour laquelle la proportion de pollen autre que le saule est significative (> $4 \%)$.

la fin de leur cycle de vie, elles collectent activement sur d'autres plantes telles que les arbres fruitiers. Cette hypothèse de changement en cours de phénologie est renforcée par la différence de comportement observée sur le terrain lors de la collecte des spécimens. Les femelles d'Andrena vaga quant à elles collectent systématiquement sur le saule et cessent toute activité de visite florale lors de la fin de floraison de leur plante hôte. Nos analyses confirment le caractère polylectique de Colletes cunicularius démontré par Müller \& Kuhlmann (2008). Les adaptations physiologiques et éthologiques liées à ces capacités de changement demeurent encore totalement inconnues, des études sont en cours.

\section{Remerciements}

Un grand merci aux personnes qui nous ont aidées dans le cadre de cette étude : L. Blondiau, L. Crepin, A. Chyzy, T. De Meulemeester et T. Lecocq (Université de Mons) ainsi qu'à C. Delcourt qui s'est chargée de l'identification des pollens (CARI asbl). Merci également à P. Rasmont (Université de Mons), N. Vereecken (Université Libre de Bruxelles) et à K. Denis (Université de Mons) d'avoir accepté de relire et corriger cet article. Enfin, la collecte du matériel a été permise grâce à l'autorisation de $\mathrm{D}$. Bauwens, responsable de la gestion de la réserve naturelle de la grande bruyère.

\section{Références bibliographiques}

Barone R, 1999. Evaluation faunistique et floristique de la Grande Bruyère de Blaton (Belgique, Hainaut). Université de Mons- Hainaut, 72 p.

Bischoff I, Feltgen $K$ \& Breckner D, 2003. Foraging strategy and pollen references of Andrena vaga (PANZER) and Colletes cunicularius (L.) (Hymenoptera: Apidae). Journal of Hymenoptera Research 12: 220-237.

Cane JH \& Sipes SD, 2006. Characterizing floral specialization by bees: analytical methods and a revised lexicon for oligolecty. Dans $\mathrm{M}$ Waser \& J Ollerton, Specialization and generalization in plant-pollinator interactions, University of Chicago Press, Chicago, pp. 99-122.

Larkin LL, Neff JL \& Simpson BB, 2008. The evolution of a pollen diet: host choice and diet breadth of Andrena bees (Hymenoptera: Andrenidae). Apidologie 39: 133-145.
Mader D, 1999. Geologische und biologische Entomoloökologie der rezenten Seidenbiene Colletes. Entomoökologie der Nestbauten und Nistsubstrate der Seidenbiene Colletes daviesanus und anderer rezenter solitärer Wildbienen und Wespen in Buntsandstein, Rotliegend, Keuper, Lias, Dogger, Tertiär und Quartär. Band 1. Logabook, Köln, xliii + 807 pp.

Mahé G, 2009. Les abeilles du genre Colletes (Hymenoptera, Colletidae) en Presqu'île guérandaise. OSMIA 3: 6-10.

Malyshev SI, 1936. The nesting habits of solitary bees. A comparative study. Eos 11: 201-309.

Michener CD, 2007. The bees of the world, second edition. Johns Hopkins University Press, Baltimore, 913 p.

Michez D, Joris I \& Iserbyt S, 2008. Eco-éthologie des visiteurs de Lythrum salicaria L. (Lythraceae) en Belgique. Belgian Fournal of Entomology 10: 37-55.

Müller A, 1996. Host-plant specialization in Western Palearctic anthidiine bees (Hymenoptera: Apoidea: Megachilidae). Ecological Monographs 66: 235-257.

Müller A \& Kuhlmann M, 2008. Pollen hosts of western palaearctic bees of the genus Colletes (Hymenoptera: Colletidae): the Asteraceae paradox. Biological Fournal of the Linnean Society 95: 719-733.

Ponchau O, Iserbyt S, Verhaeghe J-C \& Rasmont P, 2006. Is the caste ratio of the oligolectic bumblebee Bombus gerstaecker Morawitz (Hymenoptera: Apidae) biased to queens? Annales de la Société entomologique de France (n.s.) 42: 207-214.

Rasmont P, 1988. Monographie écologique et biogéographique des bourdons de France et de Belgique (Hymenoptera, Apidae, Bombinae). Faculté Universitaire des Sciences Agronomiques de Gembloux, Gembloux, 309 + LXII p.

Robertson C, 1925. Heterotrophic bees. Ecology 6: 412-436.

Vereecken N, Toffin E, Gosselin M \& Michez D, 2006. Observations relatives à la biologie et à la nidification de quelques abeilles sauvages psammophiles d'intérêts en Wallonie 1. Observations printanières. Parcs et réserves 61: 8-13.

Waser NM \& Ollerton J, 2006. Plant-Pollinator Interactions - From Generalization to Specialisation. The University of Chicago Press, Chicago, 445p.

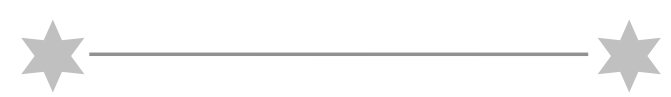

